\title{
Advanced Art Authentication in Oil Paintings Using Precise 3D Morphological Analysis of Craquelure Patterns
}

\section{Soojung Kim}

Pusan National University

\section{Sang Min Park}

Pusan National University

\section{Seongjin Bak}

Pusan National University

Gyeong Hun Kim

Pusan National University

\section{Chang-Seok Kim}

Pusan National University

\section{Chang Eun Kim}

Pusan National University

Kyujung Kim ( $\nabla$ k.kim@pusan.ac.kr)

Pusan National University

\section{Research Article}

Keywords: art authentication, morphology, optical coherence tomography (OCT)

Posted Date: June 24th, 2021

DOl: https://doi.org/10.21203/rs.3.rs-583044/v1

License: (a) This work is licensed under a Creative Commons Attribution 4.0 International License. Read Full License 


\section{Abstract}

The development of scientific technology for art authentication have elicited multidimensional evidence to distinguish forgeries from original artworks. Here, we analyzed three-dimensional morphology of cracks that contain information such as painting features of artworks using an optical coherence tomography (OCT). The forgeries were produced by an expert from the original oil paintings with cracks that occur as paint drying, canvas aging, and physical damage. The parameters such as shape, width, and depth were compared based on cross-sectional images of original and fake cracks. The shapes of original cracks were a rectangular and an inverted triangle, but fake cracks were a relatively simple inverted triangle. The original cracks were as deep as the thickness of the upper layer and mostly have 'thin/deep' or 'wide/shallow'. The fake cracks were observed as 'thin/shallow' or 'wide/deep'. This study will improve the understanding of the crack characteristics and promote development of techniques for art authenticity.

\section{Introduction}

The volume and value of transactions in the international art market have been increasing over the years $[1,2,3]$. General interest in artworks has progressively increased in recognition of their financial and cultural value $[4,5]$. At the same time, art forgery scandals have occurred for the purpose of monetary gain. It causes a disheartening reduction in the reliability of the global art market and economic turbulence may take place in the global art sales [6]. To sustain the artistic activities of artists and transactions in the art market, it is important to establish a specialized system for distinguishing between authentic and forged artworks. There are two methods to discriminate counterfeit works: a judging process with expert insight by observations with the naked eye [7] and scientific assessments using analytical techniques $[8,9,10]$. First, the appearance of artworks is visually inspected for painting, aging, canvas aging, and cracking generated on the surface of artworks. Standards of evaluation using this method can be subjective and limited to observational evidence in the artworks. In the second method, scientific techniques are used to examine detailed information about the artworks. For example, microscopic images based on bright-field lighting are commonly used to observe painting surfaces at high lateral resolutions ( 1,000 X) [11]. In addition, the high-end performance capabilities of electron microscopes provide useful morphological features such as surface topography and composition of art pigments though scanning electron microscope (SEM) [12] and transmission electron microscope (TEM) [13]. Infrared [14, 15] and ultraviolet [16] optical technologies can collect hidden information in the layers of undercoat paint as positive proof for artwork authentication. However, counterfeit technologies have developed rapidly and there are cases where authentication test cannot be judged to be reliable even when using various technologies. To overcome this challenge, it is necessary to develop highly sophisticated analysis techniques to improve the performance and accuracy of the artwork authentication process.

Recently, cracks have attracted considerable attention from researchers developing innovative methods in the application of artwork authentication $[17,18]$. Crack patterns are created on painted surfaces by 
environmental impact during the manufacturing process and the characteristics of painting as various artistic methods [19]. Previous studies found that cracks contain information on environmental factors, the proportions of pigments, humidity, and the unique characteristics of the artist's painting technique, making it possibility to judge the authenticity of artworks [20]. If the analytical techniques can discriminate between natural and fake cracks, it would be possible to obtain significant information on the authenticity of the artworks. As various methods of analyzing cracks, physical [17,21,22] and visual $[20,23]$ characteristics of cracks on the painted surface have been investigated. When studying the physical characteristics of the cracks, it is occasionally necessary to extract some of the pigment layers of the artworks, the internal and external structures of paintings cannot be examined physically without irrevocable damage to the original piece. On the other hand, important information can be found through a visual analysis, such as the characteristics of the painting and the types of cracks, because the geometrical properties of the cracks depend on factors that contribute to crack generation and propagation. $[9,21]$.

To analyze the visual properties of cracks, the overall shape of the crack in the top-view can be detected with the naked eye and is extremely enlarged utilizing a microscope that provides high-magnification images. However, crack patterns appear in artworks along both horizontal and vertical axes over time. Therefore, it is not possible to analyze the properties of cracks completely using the method described above, moreover, the information that can be obtained is limited. To overcome these limitations, we obtained three-dimensional shapes and cross-sectional images of cracks on a painted surface using an optical coherence tomography (OCT) system. The OCT system has been applied on height measurements on the signal intensity of two-dimensional (2D) and three-dimensional (3D) images for an accurate estimation of the crack dimension (width, depth, and morphology) $[24,25,26]$. In addition, OCT laser technology has been utilized to investigate the pigment layers of artworks without inflicting damage and has proven to be highly effective for artwork analysis [27, 28, 29, 30, 31, 32]. Cracks that occur on oil painting are more likely to be caused by external environmental factors and internal chemical changes than in other types of art [22]. To provide a basis for authenticity through crack analysis, natural cracks in oil paintings were compared with artificial cracks forged by counterfeiters through OCT. Finally, we investigated the difference between the original cracks and the forged cracks through OCT for art authentication.

\section{Materials And Methods}

\subsection{Experimental set-up}

The OCT system was constructed based on a Michelson interferometer with a beam splitter. In this setting, a spectral domain OCT (SD-OCT) system was selected for high-speed of data acquisition (Figure. S1). The developed OCT system generated a circularly polarized laser operating at a wavelength of 930 $\mathrm{nm}$. The penetration depth of this laser on the painted surface of artworks could be reduced than longer wavelength of the laser source, but the OCT system using short wavelength of the laser $(930 \mathrm{~nm})$ had a higher imaging performance with a better resolution for accurate crack detection. In this study, the 
experimental OCT system could produce an excellent longitudinal resolution of $8 \mu \mathrm{m}$ and a lateral resolution of $7 \mu \mathrm{m}$ in air and a measuring depth range of $1.7 \mathrm{~mm}$ from the surface. After laser emissions with a wavelength of $930 \mathrm{~nm}$ are transmit through the beam splitter, they are reflected from two scanning mirrors and injected onto the surface of the artwork with an objective lens. Interference signals are produced by combining the reflected optical signals from the artwork surface with the reference signals and generate 3D images that have accurate dimensional and morphological characteristics of the surface cracks. The artwork is fixed on a well-aligned $X Y$ axis stage. The XY-axis movement system can quickly and precisely adjust the position to be measured in units of several micrometer without direct contact with the artwork. The temperature of the measurement and storage area was set to $25{ }^{\circ} \mathrm{C}$ and the humidity was set to $40 \%$ to minimize damage to the artwork by environmental factors. To examine variations in the depths and shapes of the longitudinal cracks, we collected cross-sectional images to emphasize the crack morphologies exposed on the complicated surface microstructures.

\subsection{Preparation of original and forged artworks}

Three representative works were selected from 34 oil paintings and OCT analysis data were presented for this study. To analyze the general characteristics of the cracks generated in the oil paintings, artworks by artists who specialize in oil paintings were selected. Because cracks are likely to occur in old works, art works that were at least 30 years old were selected. One of the selected works was "figure" $(45 \mathrm{~cm} \times 38$ $\mathrm{cm})$, oil painting by Joon Ja Jun produced in 1964. Joon Ja Jun is an acclaimed contemporary Korean artist who was selected as a recommended artist for the National Exhibition and won three special awards and 13 awards at the Korean National Art Exhibition held from 1949 to 1981. A major theme of her work is humans and festivals. By making full use of intense gesture drawing and action painting, the artist pursues a fundamental inquiry into painting.

To compare the OCT data characteristics of natural and artificial cracks, three counterfeit artworks were produced with the consent of this writer. An art restoration specialist used appropriate oil paints and media to fully replicate the colors and cracks as accurately as possible. The cracks were artificially created by drawing them on the surface of the artwork with a physical force using various sharp knife and the thickness of the cracks was adjusted by adjusting the force. Although the result was not exactly the same as the original crack, the thickness of the painted layers and the composition of the medium were manipulated to produce counterfeit cracks similar to naturally occurring cracks. The cracks that show the remarkable characteristics of the painted layers of original and forged artworks were selected as measurement areas for OCT, respectively. Based on the OCT images of each crack, the geometric features of natural and artificial crack were analyzed.

\section{Results}

A schematic diagram depicts the authenticity assessment process for artworks based on OCT technology (Figure 1). Three artworks were selected for the OCT analysis according to the time of the production, the artist's expertise, and the characteristics of the cracks. Thirty oil paintings were leased for this research. The three artworks were oil painting by Korean artists who agreed with the contents of this study. Two of 
the artworks were produced by Eunyeong Ko who specializes in oil painting and art restoration and the third painting was produced by Joon Ja Jun who works in oil. Natural cracks occur due to various causes such as physical and chemical reactions at the painted surface for each work, and these cracks were analyzed using OCT technology (Figure 1a). The art restoration expert, who created two of the artworks, produced forgery works for each of his two artworks and one of other artworks with the permission of the original artist. The art restoration expert could accurately understand the color combination of the original work, the thickness of the paint layer, the texture of the surface, and the painting style because these abilities are essential for restoring damaged areas in artworks. Therefore, it was difficult to differentiate between the original works and the forged works produced by professional forgery methods. Specifically, by replicating the color combination of the paints and the thickness of the paint layer, the forged artworks had similar paint techniques to the original works. In addition, forged cracks with the same directions, thickness, and positions to the natural cracks in the original works were artificially created in the forged artworks (Figure 1b).

Before analyzing the paintings with OCT technology, several cracks that represent he characteristics of the artworks were selected from among several cracks in each original work. In the counterfeit works, the artificial cracks, made in locations similar to those of the selected original work, were selected. The OCT scanning was performed by selecting a measurement area of $2 \mathrm{~mm} \times 2 \mathrm{~mm} \times 1.7 \mathrm{~mm}$ in the crack position at the artwork surface (Figure 1c and d). Next the 3D modeling data containing information on the depth, width, cross section, and shape of the cracks were obtained, and a cross-sectional image in the middle plane of the cracks was extracted as representative for the quantitative analysis of crack morphologies. In the cross-sectional image, various parameters such as width, depth, and shape were measured according to the morphological diversity and complexity of the cracks. In particular, the width and depth were subdivided into three, which was intended to analyze the crack characteristics in detail as much as possible (Figure 1e and $\mathbf{f}$ ). Based on the data obtained in this way, the characteristics of the cracks were classified and analyzed according to the cause of the crack, and the foundation for the authenticity of the artwork was established. In addition to the studies selected, various oil paints were analyzed, and a quantitative analysis of the OCT cross-sectional images and crack parameters was conducted. These works were also original works, and focused on analyzing the characteristics of cracks that occurred in various works of art. This analysis was referred to when analyzing the cracks in this study (Figure S2-11).

\subsection{Oil paintings and forged works prepared for 3D analysis of cracks based on the OCT system}

To analyze the differences between the original and forged cracks on the artworks, we obtained three oil paintings created on canvas by two professional artists (Figure $\mathbf{2 a}, \mathbf{b}$ and $\mathbf{c}$ ). With the permission of the original artist, the restoration expert produced counterfeit works of the original works using the appropriate oil paint and medium after observing the style, color, and cracks of the original work through the naked eye and a magnifying lens (Figure $\mathbf{2 d}$, e and $\mathbf{f}$ ). The forged cracks on the counterfeit works were reproduced through common methods of creating fake cracks, such as knife tools. The main cracks that reveal the characteristics of the paint layer in the real work were similarly produced in counterfeits. The 
geometric characteristics of natural and artificial cracks were analyzed, based on the OCT image appearing in each crack.

Artwork 1: Figure 2a shows the oil painting with light brown and darker brown upper layers on top of a light green undercoat layer applied to a canvas. Cracks occur in both the light brown and darker brown upper layers, except for the under layer of paint. This also occurred because the amount of the painting medium in the upper paint layers was not adequately controlled.

Artwork 2: In Figure 2b, the artwork has multiple layers composed of a black underpainting layer and upper layers with a mixture of white, light blue, and green colors. The cracks occurred on the upper layers because the upper painted layers contained relatively little medium.

Artwork 3: We obtained an oil painting produced by Joon Ja Jun in 1964 (Figure 2c). The title of this artwork is "figure" (45 cm $\times 38 \mathrm{~cm})$. In this work, the artist attempted to overlay the surface of the artwork with light purple paint to cover the cracks occurred naturally in the work. This work was selected for art authentication based on OCT analysis because of occurrence of various crack types in paint layers with varied thicknesses. Because the new canvas has good elasticity and does not crack easily, a canvas that was produced approximately 25 years ago was used as the substrate of the forged artwork. In addition, the thickness of the paint layer on the forged artwork was similarly drawn by observing the original product with the naked eye (Figure 2f).

\subsection{The appearance and location of the cracks selected to measure OCT system in original works and forgery works painted by oil painting}

Target regions for the OCT analysis were selected at three locations where cracks were generated in each work. The three selected points represented crack locations that were expected to provide particularly meaningful information for art authentication based on the OCT analysis of cracks.

Artwork 1: In Figure 3a, cracks occurred because the proportion of oil painting mediums used in the upper paint layers (light brown/dark brown) was not adequately controlled like the conditions of optimized combinations for preventing cracks in the painting. Forged cracks were produced in both the light and dark brown paint layers to match the locations at which the original cracks were generated. The cracks were produced by drawing thin lines similar to cracks using sharp knives on the paint layers with detailed control so that the color on the bottom side of the paint layer was visible (Figure $3 \mathbf{b}$ ).

Artwork 2: In Figure 3c, the under layer of the artwork was painted black, and the colors of the upper layer were composed of a mixture of white, light blue, and light green paint. Unlike Artwork 1, cracks were generated because there was little paint medium in both the upper and under layers. Some cracks were relatively thin so that the color of the underlayer was not visible. Although the location of the cracks in the forged work was not similar to the location of the original cracks, the forged cracks were made so that it was difficult to distinguish between the cracks with the naked eye (Figure $3 \mathbf{d}$ ). In addition, the thickness of the forged cracks was controlled to be as similar to the original as possible. 


\subsection{Comparison of cross-sectional images of the real cracks and the counterfeit cracks analyzed by using the OCT system}

The cracks created in the original and counterfeit artworks were measured using the OCT system, and cross-sectional images of the cracks were compared to analyze the differences in the morphologies of the original and forged cracks (Figure 4). The images were extracted from the 3D images of the crack and the cross-sectional images of natural and counterfeit cracks were compared to examine the differences in the morphologies, such as the depth and shape. These images contain adequate information because they can clearly identify the 3D visual features of the crack that cannot be observed by the naked eye or by a microscope.

Artwork 1 (Figure 4a): In Figure 4, the cracks caused by the combination of media occur for each color, but the shape of the cracks for each color was not significantly different. The most prominent feature in the OCT cross-sectional image of the original cracks in Artwork 1 is a rectangular shape in which the side interface of the crack is shaped like a cliff and the bottom surface of the cracks is flat. This means that when the mediums in the upper painted layer dried, they left many gaps in the paint, resulting in a complete loss of internal adhesion. As the upper layer dried, the internal bonding decreased, which is why the side of the crack was clean. However, the surface of the forged cracks, such as surface roughness, tended to be uneven. It appeared similar on the top side, but there was a clear difference in the OCT crosssectional images.

Artwork 2 (Figure 4b): The lower layer of paint had more medium than the upper layer, and as the medium solutions dried, small empty pores were created inside the space of the lower painted layer. As a result, the adhesion inside the lower painted layer decreased and this phenomenon seems to have affected the occurrence of cracks in the upper layer of paint. The main characteristic of the cracks is that the width of the cracks is very thin, so that the color of the lower layer is not visible, and the aspect ratio (depth/width) of the cracks was quite large. The original cracks had thin and deep rectangular shapes, but the counterfeit cracks had triangular shapes with rough surfaces. A concentrated force was applied with knives to create counterfeit cracks with the thin width similar to that of original cracks. As a result, the counterfeit cracks appeared to be relatively shallow in depth.

\subsection{Quantitative analysis of an OCT cross-section image of original and forged cracks of Artworks 1 and Artwork 2}

The widths (top width, center width and bottom width) and depths (left depth, center depth, and right depth) of each crack were quantified using cross-sectional image analysis. Figure $\mathbf{5}$ shows the morphology of the cracks formed in the forgery and original works of art, and the quantitatively measured values of the scale parameters of each crack were classified into various types. These representative values were selected from the largest values of the crack widths and depths and were used to compare the original artworks and counterfeit artworks (Figure 5b-d). 
Artwork 1: The cracks of the original artworks have a rectangular shape, so they were measured equally for each measurement parameter of the crack width. On the other hand, the cracks in the forged artworks have inverted triangular shapes and the measured values were different for each parameter type, and the bottom parameter values were not measured. The depth of cracks in counterfeit artworks and original

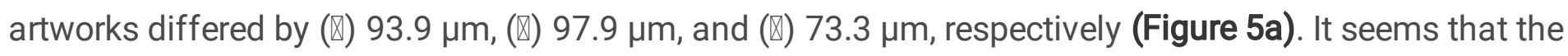
depth of the crack increased as the force is applied to the painting layer with a knife to make the original cracks similar in width.

Artwork 2: The medium combination problem occurs in the lower paint layer. The depth value was observed to be approximately 2.5 times larger than the width of the crack (Figure $5 \mathrm{~b}$ ). In the case of thin and deep cracks, the cross-sectional shape of the crack was either a thin rectangular or an inverted triangle shape. The weakened bonding force in the lower paint layer affected the upper layer, and it was judged that the crack at the upper part occurred in a thin width.

\subsection{Location and enlarged images for a comparison of original and forged cracks in Artworks 3}

To analyze the original cracks created by other factors and the counterfeit cracks, we borrowed oil paintings on canvas produced by Joon Ja Jun. Five cracks were classified into five types according to the characteristics of the original artworks and the cause of cracks occurrence. The forged cracks were produced by scratching with knives and stabbing with pointed tools and broadly similar to techniques used to counterfeit artworks in the real world.

Craquelure $\mathbb{\bigotimes}:$ These cracked areas occurred due to aging and shrinkage of the canvas at the paint layers that are thinner than the others (Figure 6a' $\mathbb{X}$ ). This speculative rationale is that wrinkles appeared in the same direction on the back of the canvas as the cracks advanced on the painted surface. These cracks occurred in locations painted with various pigments, regardless of the colors of the paint layers. To imitate the visual morphology of the original crack, thin mass tools were used by finely tuned slash to create long paths of the dynamic cracks in the painting layer of the forged work (Figure $\mathbf{6} \mathbf{b}^{\mathbf{}} \boldsymbol{\bigotimes}$ ). As a result, counterfeit cracks with thicknesses and directions similar to those of the original cracks were created.

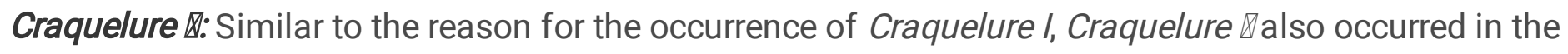

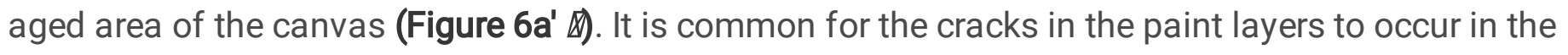
direction of the thick wrinkles on the back of the canvas, but the difference is that the thickness of the

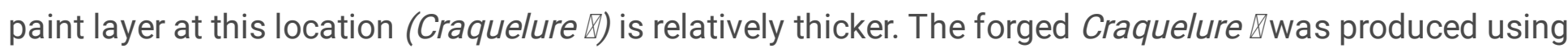
the same slashing method used to create forged Craquelure I (Figure 6 $\left.\mathbf{b}^{\prime} \bigotimes\right)$.

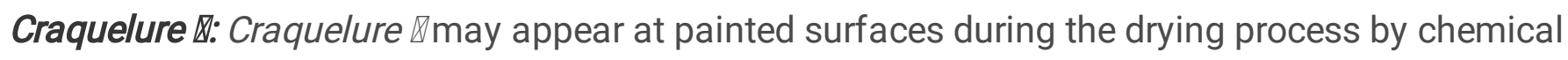
environmental changes in the layers of paint (Figure 6a' $\rrbracket$ ). The cracks were generated across painted layers with the mixture of three colors that were applied thicker than the surrounding layers of paint. In the middle of the paint layer, it seems that painting mediums were poorly mixed, and this led to the drying cracks occurring in large areas of the painted surface. Forged cracks were also produced in a pale-yellow 
color of an undercoated layer through a deliberate process in which the force was appropriately adjusted with a thin knife (Figure 6b' 囚).

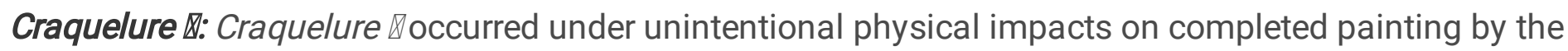
artist and appeared to be scratched by an ambiguous tool (Figure 6a' $囚$ ). An ivory color of a lower layer of paint was visible in an exposed area. During the drying process, the width of the cracks widened with the formation of many branches in the cracks. When these cracks were forged, they were created by applying deliberate force to the painted surface using a stainless-steel tool. The width of the forged crack was suitably widened, and the lower surface layer was clearly visible, similar to the original cracks (Figure $6 b^{\prime} \otimes$.

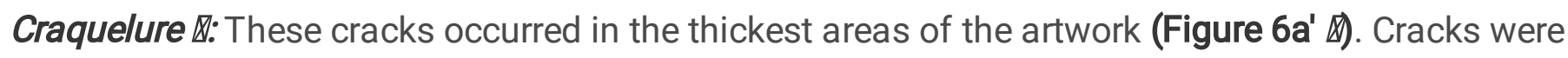
observed across both the upper layer (white color) and a sublayer (blue color). To replicate the original cracks, a thicker knife was used to apply a relatively stronger force to the painted surface layer (Figure $6 b^{\prime} \rrbracket$.

\subsection{Visual comparison based on the OCT cross-sectional images of cracks selected from an original and a fake artwork.}

Craquelure 甘: The cross-sectional shape of the original Craquelure thas an inverted triangular shape in the upper painted layer (Figure 7a $\searrow$ ). The shape is formed into a sharp point toward the undercoated layers, and the height of both side layers along the crack remains approximately constant without protruding outer layers. The upper painted layers were affected by shrinkage that occurred due to the aging of the canvas, and the cracking process may have advanced in the top layer of the paint. Thin thread-like structures are exposed in the inner side of the cracks. This may have occurred because the ratio of medium mixture to oil painting is different for each colored layer and result in uncompleted separation inside the painted layers. Likewise, the cross-sectional morphology of the forged cracks

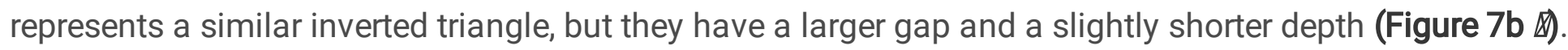
The outer surface of the forged cracks appears only on one side of the protrusion phenomenon of the painted layers because of the physical influence of drawing the crack lines.

Craquelure $\mathbb{X}$ : The crack formation has an inverted triangle with a slight incline (Figure 7a $\bigotimes$ ). The width of

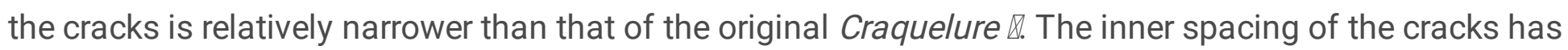
an uneven structure without a thread-like fiber. In the cross-sectional image of the counterfeit cracks, one side of the painted layers has a slightly overhung outer painted surface (Figure $\mathbf{7 b} \mathbb{\mathbf { b }}$ ). The forged cracks have a relatively shallow depth, and the sidewalls of the cracks are neat similar to the forged Craquelure प.

Craquelure $\mathbb{\mathbb { V }}$ : The cross-sectional shape of the crack shows an inverted triangle with a tip that has a relatively blunt shape (Figure 7a $\mathbb{Z}$ ). The central axis of the inverted triangle is slightly inclined. The thin width of the counterfeit cracks was produced to reveal the color of the underlying paint layer, like the original cracks (Figure $7 \mathrm{~b} \otimes$ ). Although the widths of the two cracks appear similar to each other, the 
foremost difference between these two cracks can be accurately distinguished according to the depth and shape in only the OCT cross-sectional images.

Craquelure $\mathbb{\mathbb { V }}$ : Cracks with a large width are visible on the top of the outer painted layer, and a sharply thin

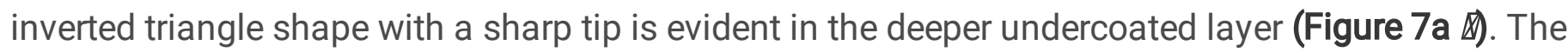
forging process was conducted under circumstances where the coating thickness is not exactly known. These forged cracks were produced to reveal the ivory-color of the under layer similar to the original cracks, but the difference in depth and shape is evident in the OCT cross-sectional image (Figure $7 \mathbf{b} \bigotimes$ ).

Craquelure $\mathbb{0}:$ The sidewall of the cracks appears to be relatively neat, as the painted layer appears to have dried the most (Figure 7a $\bigotimes$ ). The original Craquelure \has a wide crack width, but the color of the lower layer is not visible. Because the paint layer is thick, the crack is not deep enough to reveal the color

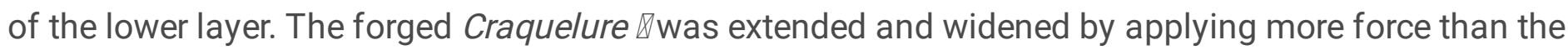
process of producing other forged cracks The sidewall of the cracks appears to be relatively neat, as the painted layer appears to have dried the most (Figure $\mathbf{7 b} \mathbb{\Delta}$ ). Therefore, the upper layers were extruded from the edges of the cracks. In addition, the side of the forged crack was neatly made in a straight line, but the detailed appearance with a zigzag shape from the paint layer of the original crack could not be forged.

\subsection{Quantitative analysis of OCT cross-section image of original and forged cracks of Artworks 3}

Craquelure $\mathbb{\mathbb { V }}$ : The natural cracks were generated by canvas shrinkage and are $70.2 \mu \mathrm{m}$ in width and 370.2

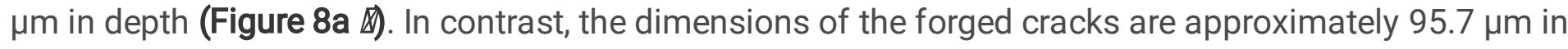
width and $142.5 \mu \mathrm{m}$ in depth. This observation may indicate that forged cracks have been a wider and shallower crack spacing than the original cracks. There is a statistically significant difference of about $227.7 \mu \mathrm{m}$ in crack depth between the original and forged cracks. Although the force was finely adjusted to create the thin width, it was almost impossible to control the depth with any precision.

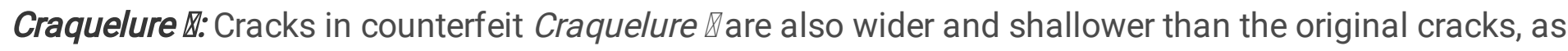

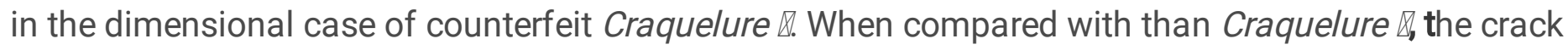
widths are relatively thinner by a difference of $20 \mu \mathrm{m}$, but the depth is shallower with a difference of 134 $\mu \mathrm{m}$ (Figure 8a $\bigotimes$ ). The original Craquelure $\nabla$ and $\square$ were created by canvas aging and Craquelure // occurred in thicker paint layers than in Craquelure $\mathbb{Q}$. However, it is very difficult to counterfeit cracks according to tendency of natural crack shapes, produced according to the thickness of the paint layer. As a result, the shapes and dimensions of the original Craquelure $\nabla$ and $\square$ are different, but forged Craquelure $\nabla$ and $\square$ have similar shapes, widths, and depths.

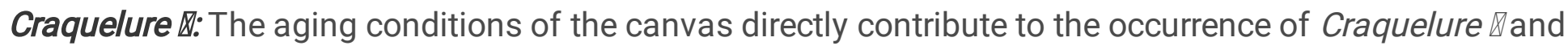

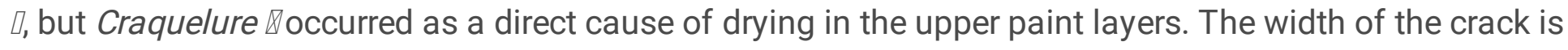
approximately $89.4 \mu \mathrm{m}$, showing a relatively wider appearance. Conversely, the depth is relatively shallow at $180.9 \mu \mathrm{m}$ (Figure $8 \mathbf{a} \bigotimes$ ). The Craquelure $\nabla$ and $\nabla$ were produced by the influence of shrinkage in the

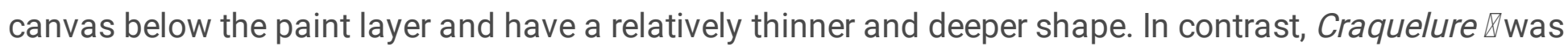


caused by the drying of the paint layer and is represented a reverse shape with a relatively broad width and shallow depth.

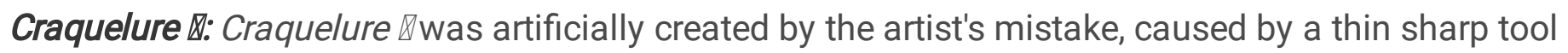
scraping the artwork's surface. The original cracks show a larger upper width with the shape of an inverted triangle, and the counterfeit cracks show a large bottom width. The original crack was approximately $191.5 \mu \mathrm{m}$ in depth, which is greater than the forged crack depth of $68.0 \mu \mathrm{m}$ (Figure $8 \mathrm{a} \triangle$ ). It may resemble that original cracks were initially scratched, and after considerable time, the cracks deformed in width and depth under paint conditions such as paint drying. In addition, the width/depth ratio of the original cracks and the forged cracks have similar dimensions.

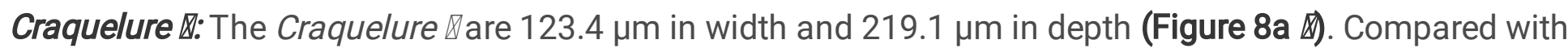
the Craquelure $\otimes$ that occurred in the thinner paint layer, the width in Craquelure $\nabla$ was wider by $34.0 \mu \mathrm{m}$

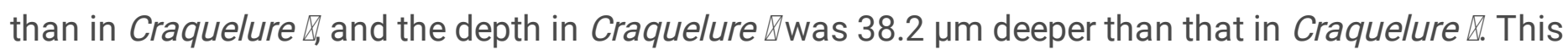
was observed in the opposite tendency of the original Craquelure $\triangle$ and $\square$, which were affected by the canvas underneath the painted layers. If natural cracks are directly influenced by the condition inside the upper painted layers, the thicker painted layers generate cracks with a greater width and depth at the painted surface. On the contrary, if cracks occur because of the influence of the canvas layer, the thicker the paint layer, the thinner and shallower the crack. Forged cracks were $68.1 \mu \mathrm{m}$ in width and $104.3 \mu \mathrm{m}$ in depth. Various cracks with similar widths can be artificially produced, but the depth is difficult to control and not as deep as the original cracks (Figure $\mathbf{8 b - d )}$.

\section{Discussion}

Cracks in works of art occur for three reasons: the drying of the paint layer, factors of related to canvas aging, and direct physical impacts on the painted layer. The cracks were measured through OCT analysis. First, cracks occurred in all three works owing to the drying of the painted layers. In Artwork 1, wide cracks occurred in the upper layers that contained a larger amount of medium than the lower layer. On the other hand, thin cracks indirectly occurred in the upper layers of Artwork 2 because the lower layers had more medium content than the upper layers. When drying advanced, the medium inside the painted layers evaporated, leaving many empty spaces in the painted layers. The adhesion between the painted layers decreased, resulting in cracking. If this cracking occurs mainly in the upper layer, there is a tendency for the width of the cracks to be wide, as in Artwork 1 (Fig. 4a). The entire thickness of the upper layer is split, the depth of the crack corresponds to the thickness of the upper layers. Conversely, if this cracking occurs mainly in the lower layers, the reduced adhesion in the lower layers affects the upper layers. Thin and deep rectangular cracks can be observed in the upper layers of Artwork 2 (Fig. 4b). In Artwork 3, the paint layer dried over a long period of time in addition to the aging of the canvas. Unlike Artwork 1 and 2 , cracks occurred by the factors of both upper and lower layers. Unlike previous cracks, the cross-sectional shape of the inverted triangle was observed. When the paint layer was thicker, the cracks were wider and observed more deeply. 
The second factor was the aging of the canvas, which affected the paint layers and generated cracks. These cracks were observed only in Artwork 3 and occurred in the thin and thick areas within the artwork. Both were affected by canvas aging, but in the second case, cracks occurred where canvas aging occurred particularly strongly. The cause of the cracking appeared in the support under the paint layers, which was relatively lower than in the first case. The weakened adhesive force in the lower layer is applied so that the upper layers have cracks, and the thinner the thickness of the upper paint layers, the easier it is to produce cracks. Therefore, in this case, larger values were observed for the width and depth of the crack.

As a third factor, cracks occurred due to physical shock and were observed only in Artwork 3. A crack was created in the original work owing to an unintended physical impact, but a long time has since passed. The cracks in the forgery were also made by physical impact, but the difference from the original cracks was how long time has passed. The much older crack was found to be deformed wider and deeper. Analysis of these two cracks reveals how deformation occurs over time in the crack generated by physical impact. Based on the data analyzed above, it is possible to track whether a crack has been forged or whether the time lapse when the crack occurred is appropriate compared to the time of production of the work for the purpose of authenticity assessment. According to the results of the analysis, the width and depth ratio of cracks in the same work differ according to the W/D cause; therefore, it is necessary to measure several cracks and compare the width and depth ratios (Fig. 8b-d).

\section{Conclusion}

OCT technology can analyze the characteristics of natural cracks, but at the same time, find differences from forged cracks. The differences between natural cracks and forged cracks were analyzed by quantitatively measuring the parameters (width, depth, and cross-sectional shape) of the cracks. In the condition using artificial or natural methods, it was confirmed that the crack widths could be similar. The results inferred that it is not sufficient to determine the authenticity of artworks with only one crack width factor. The depth of cracks can provide a useful basis for determining the authenticity of an artwork. Natural cracks can become deep as the thickness of the upper paint layer in rectangle or inverted triangle, but artificial cracks are not made in the same way as these natural cracks. The depth of artificial cracks is not as finely controlled as that of natural cracks. Because there is a limit to controlling the depth and width of natural cracks, it is possible to analyze whether it is a fake crack or not. If the cross-sectional shape is also considered in addition to the depth, the difference between natural and artificial cracks is clearly revealed, which can further increase the possibility of success in authenticity detection. The crosssectional shape of natural cracks varies, and includes a rectangle, a trapezoid, an inverted triangle with a clean surface, an inverted triangle with an internal microstructure, and an inverted triangle with a wide width only in the upper layer. However, most artificial cracks have a similar inverted triangle shape and have two shape, such as wide/deep or narrow/shallow. The distinct difference between natural and artificial cracks according to crack parameters makes OCT technology useful for appraising the authenticity of artworks. Furthermore, by continuously measuring the surface of an art object with OCT technology and observing the development of cracks and changes in the surface, it will be possible to 
collect various data that can prevent forgery of the art object. Through this study, the difference between natural and artificial cracks in oil paintings could be analyzed using OCT technology, and further it will show a breakthrough potential in the authenticity of different type of artworks.

\section{Declarations}

\section{Conflicts of interest}

The authors declare no conflicts of interest.

\section{Acknowledgements}

This work was supported by Art Appraisal \& Authentication Infrastructure Project through the Ministry of Culture, Sports and Tourism and Korea Arts Management Service (KAMS) (KAMS, 2018-2020) and was supported by the National Research Foundation of Korea (NRF) grant funded by the Korea government (MSIT) (No. 2020R1A2C4002732).

\section{References}

1. Candela, G. \& Scorcu, A. E. A price index for art market auctions. J. Cult. Econ. 21, 175-196 (1997).

2. David, G., Oosterlinck, K. \& Szafarz, A. Art market inefficiency. Econ. Lett. 121, 23-25 (2013).

3. Renneboog, L. \& Spaenjers, C. Buying beauty: on prices and returns in the art market. Manage. Sci. 59, 36-53 (2013).

4. Mei, J. \& Moses, M. Art as an investment and the underperformance of masterpieces. Am. Econ. Rev. 92, 1656-1668 (2005).

5. Locatelli Biey, M. \& Zanola, R. Investment in paintings: A short-run price index. J. Cult. Econ. 23, 211222 (1999).

6. Fink, C., Maskus, K. E. \& Qian, Y. The economic effects of counterfeiting and piracy: A review and implications for developing countries. World. Bank. Res. Obs. 31, 1-28 (2016).

7. Willems, S. Forging reality: Surface and reductionism in the work of Dick Bengtsson. Journal of Contemporary Painting 5, 233-252 (2019).

8. Lyu, S., Rockmore, D. \& Farid, H. A digital technique for art authentication. Pro. Nati. Acd. Sci. 101, 17006-17010 (2004).

9. Hwang, S. et al. Optical measurements of paintings and the creation of an artwork database for authenticity. Plos one 12, e0171354 (2017).

10. Elgammal, A., Kang, Y. \& Leeuw, M. Den. Picasso, matisse, or a fake? Automated analysis of drawings at the stroke level for attribution and authentication. In Proceedings of the AAAI Conference on Artificial Intelligence 32, No. 1 (2018).

11. Dredge, P., Wuhrer, R. \& Phillips, M. R. Monet's painting under the microscope. Microsc. Microanal. (2003). 
12. Schreiner, M., Melcher, M. \& Uhlir, K. Scanning electron microscopy and energy dispersive analysis: applications in the field of cultural heritage. Anal. Bioanal. Chem. 387, 737-747 (2007).

13. MacDonald, B. L. et al. Hunter-gatherers harvested and heated microbial biogenic iron oxides to produce rock art pigment. Sci. Rep. 9, 1-13 (2019).

14. Smith, G. D., Nunan, E., Walker, C. \& Kushel, D. Inexpensive, near-infrared imaging of artwork using a night-vision webcam for chemistry-of-art courses. J. Chem. Educ. 86, 1382-1388 (2009).

15. Gavrilov, D. et al. Infrared methods in noninvasive inspection of artwork. International Conference on NDT of Art, number May 1-5 (2008).

16. Cosentino, A. Practical notes on ultraviolet technical photography for art examination. Conservar Patrimonio 21, 53-62 (2015).

17. Léang, M., Giorgiutti-Dauphiné, F., Lee, L. T. \& Pauchard, L. Crack opening: from colloidal systems to paintings. Soft Matter 13, 5802-5808 (2017).

18. Flores, J. Entropy Signature for crack networks in old paintings: saturation prospectus. Entropy 20, 772 (2018).

19. Karpowicz, A. A study on development of cracks on paintings. J. Am. Inst. Conserv. 29, 169-180 (1990).

20. Elkhuizen, W. S. et al. Comparison of three 3D scanning techniques for paintings, as applied to Vermeer's 'Girl with a Pearl Earring'. Heritage Science 7, (2019).

21. Krzemień, L., Łukomski, M., Bratasz, Ł., Kozłowski, R. \& Mecklenburg, M. F. Mechanism of craquelure pattern formation on panel paintings. Studies in Conservation 61, 324-330 (2016).

22. Giorgiutti-Dauphiné, F. \& Pauchard, L. Painting cracks: A way to investigate the pictorial matter. J. Appl. Phys. 120, (2016).

23. Bucklow, S. The Description of Craquelure Patterns. Studies in Conservation 42, 129-140 (1997).

24. Mariampillai, A. et al. Optimized speckle variance OCT imaging of microvasculature. Opt. Lett. 35, 1257-1259 (2010).

25. Mette, M., Lars, T., Thomas, M. J., Peter, E. A. \& Gregor, B. E. J. OCT imaging of skin cancer and other dermatological diseases. J. Biophotonics 2, 442-451 (2009).

26. Sergeev, A. et al. In vivo endoscopic OCT imaging of precancer and cancer states of human mucosa. Opt. Express 1, 432-440 (1997).

27. Liang, $\mathrm{H}$. et al. Optical coherence tomography and non-linear microscopy for paintings-a study of the complementary capabilities and laser degradation effects. Opt. Express 25, 19640-19653 (2017).

28. Gurov, I., Margaryants, N. \& Zhukova, E. Evaluation of art subjects implemented in the marquetry technique by the optical coherence microscopy method. Strain 55, e12304 (2019).

29. Latour, G., Georges, G., Siozade, L., Deumié, C. \& Echard, J.-P. Study of varnish layers with optical coherence tomography in both visible and infrared domains. O3A: Optics for Arts, Architecture, and Archaeology II 7391, 73910J (2009). 
30. Thickett, D. et al. Using non-invasive non-destructive techniques to monitor cultural heritage objects. Insight: Non-Destructive Testing and Condition Monitoring 59, 230-234 (2017).

31. Targowski, P. et al. Using Optical Coherence Tomography to Reveal the Hidden History of The Landsdowne Virgin of the Yarnwinder by Leonardo da Vinci and Studio. Angew. Chem 130, 73967400 (2018).

32. Targowski, P., Góra, M. \& Wojtkowski, M. Optical Coherence Tomography for Artwork Diagnostics. Laser Chem. (2006).

\section{Figures}

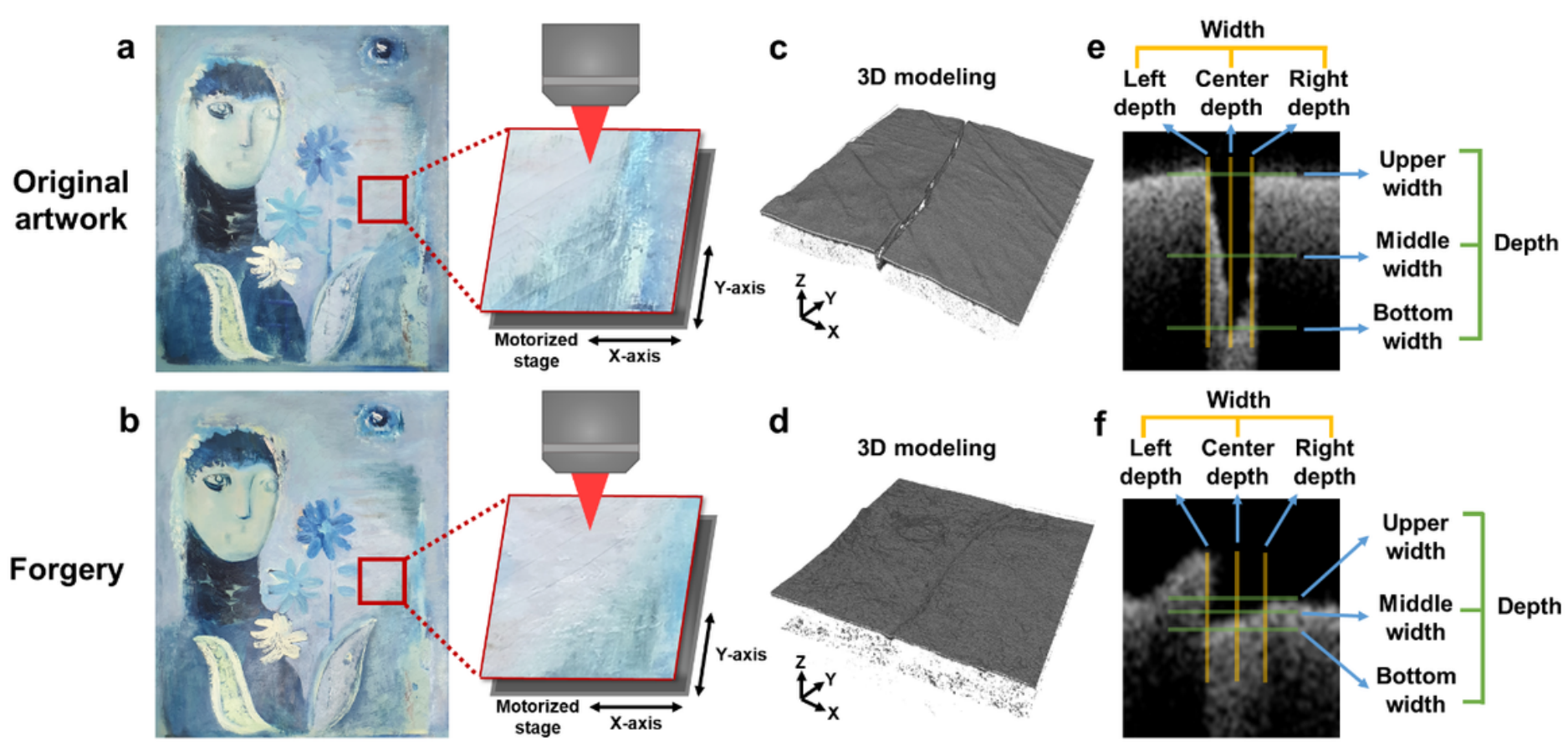

\section{Figure 1}

Schematic of the OCT system-based on the Michelson interferometer. $(a, b)$ The OCT system was set up to measure the crack area of the artwork. This system can fix artworks on the stage to prevent unwanted movement while the artworks was being measured. The stage moves them in units of at least $10 \mu \mathrm{m}$ along the $X Y$ axis and place the focused beam on the crack area to accurately measure the desired area. $(c, d) 3 D$ data of art objects were acquired, and $(e, f)$ cross-sectional images were extracted to compare the width and depth parameters of the original and forged artworks. 
Artwork 1

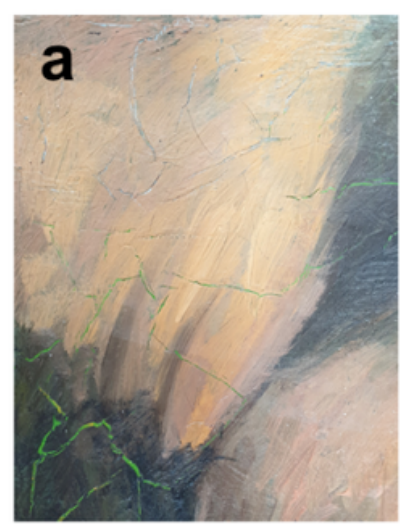

Original artworks

Forgery

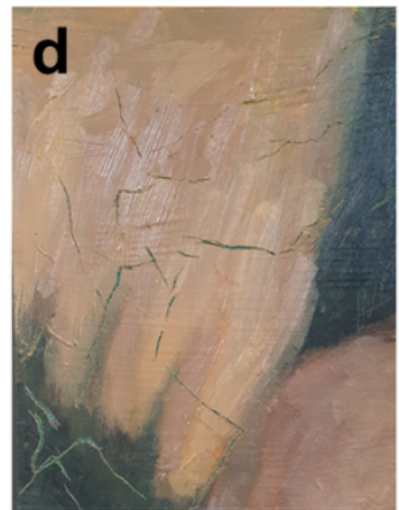

Artwork 2
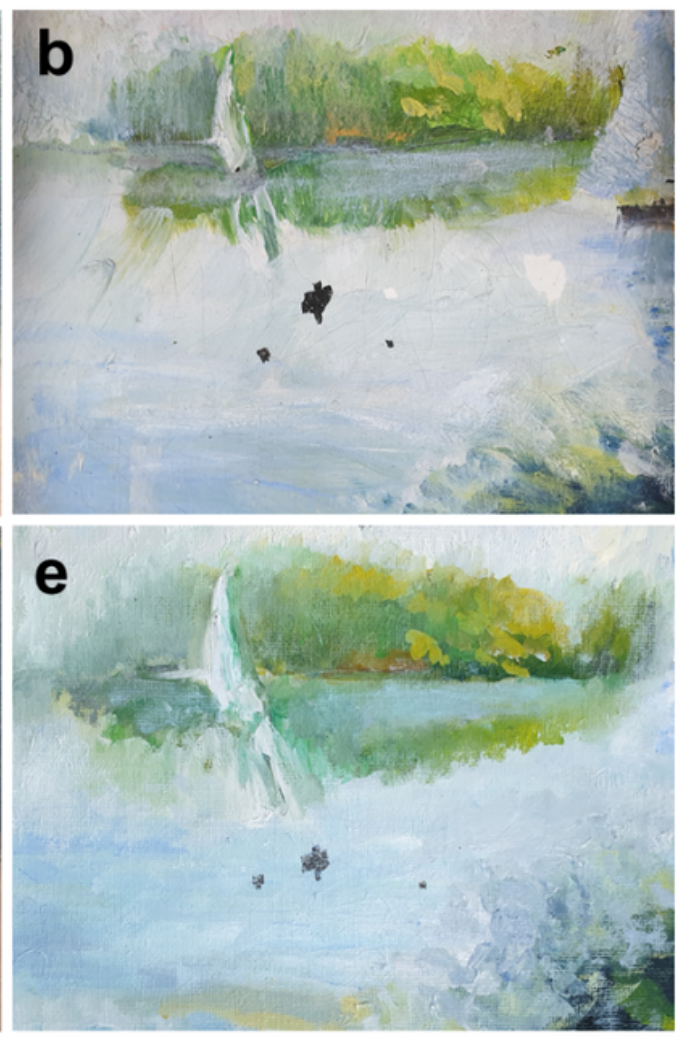

Artwork 3
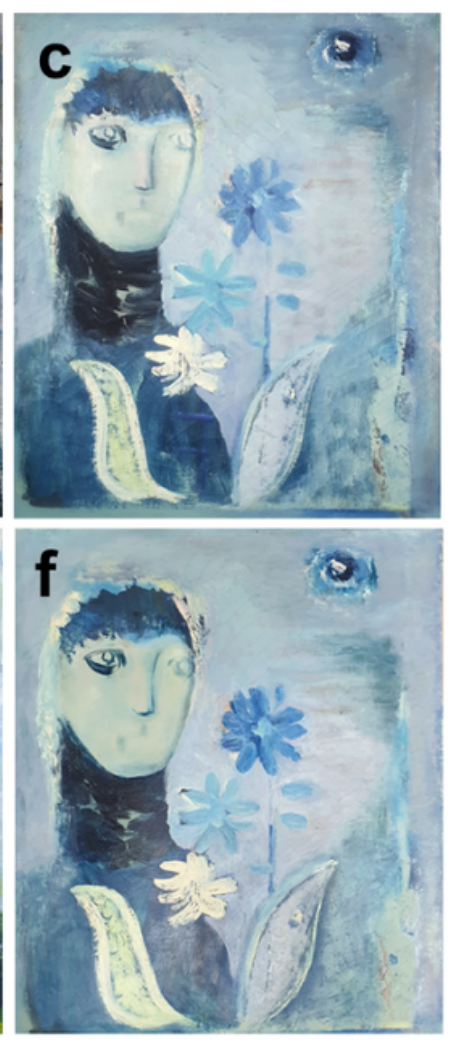

\section{Figure 2}

Original oil painting $(a, b$, and $c)$ and counterfeit works ( $d, e$, and $f)$. They were prepared for an analysis of the three-dimensional forms of cracks used for authenticity identification of art works. The forgeries were completed by checking the color combination of the original work, the artist's brushstroke style, and the thickness of the paint layer as much as possible. 
Artwork 1
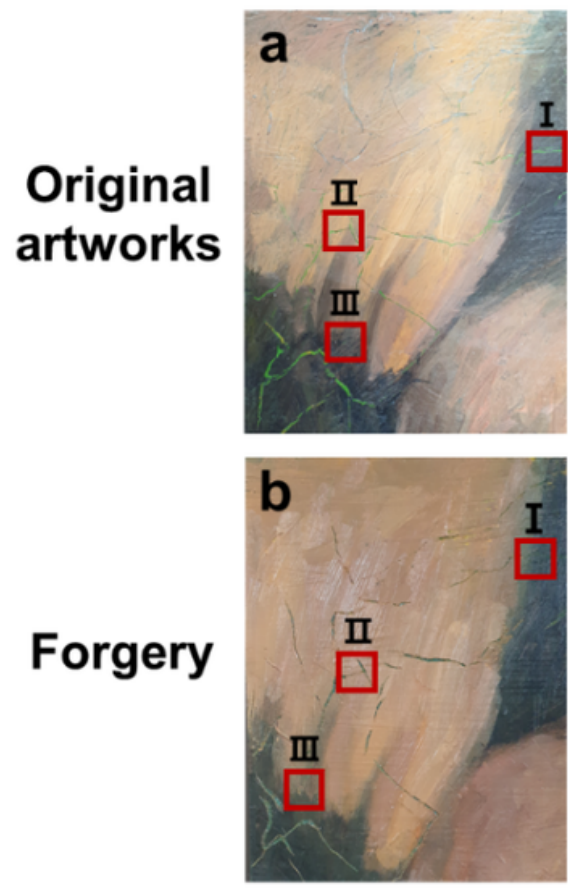

Artwork 2
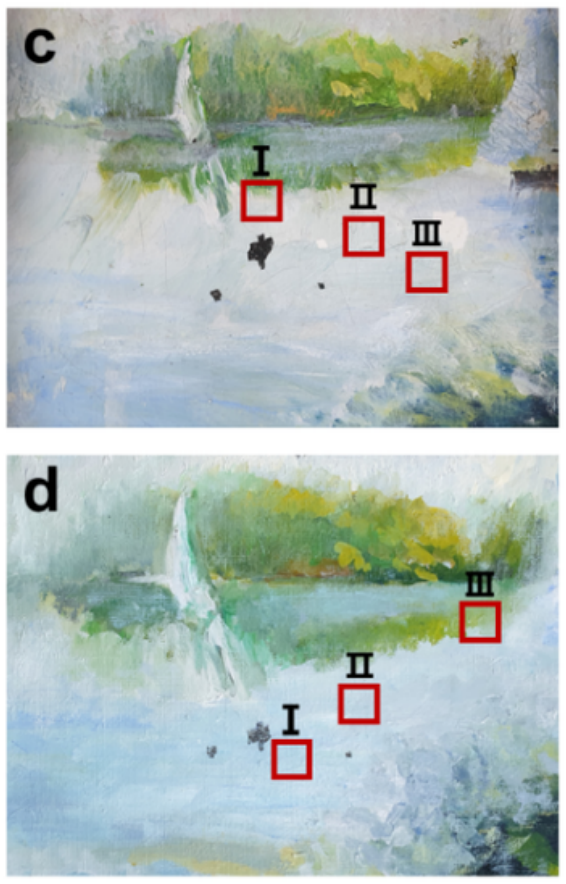
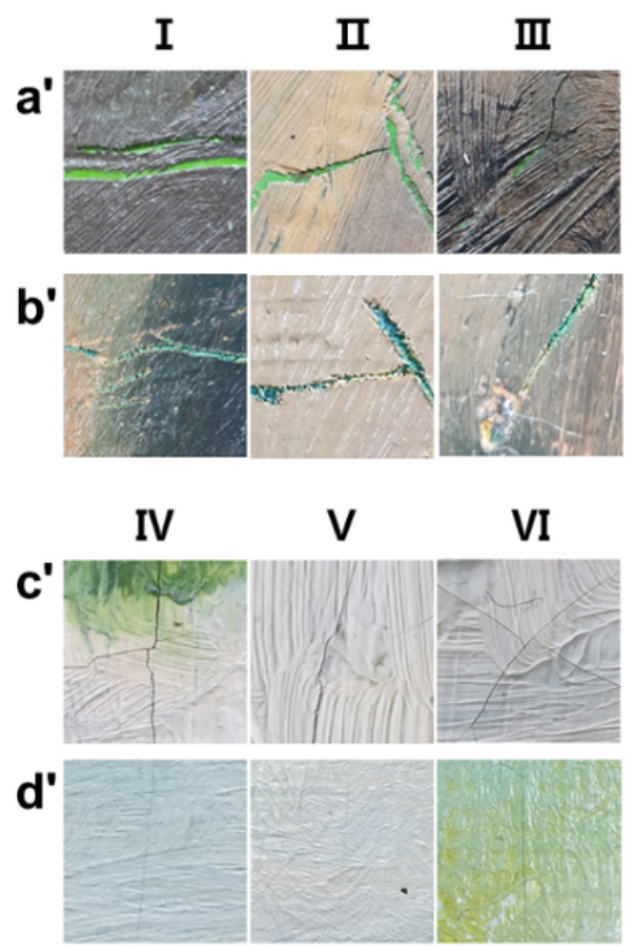

\section{Figure 3}

The artworks showing the area selected by the OCT system and enlarged views of the cracks in each area. The cracks were selected because the paint characteristics of each work were prominent. They were then analyzed with the OCT system. Enlarged images of original cracks ( $\left.a^{\prime}, b^{\prime}\right)$ and fake cracks ( $\left.c^{\prime}, d^{\prime}\right)$ at the selected location in Artwork 1 and Artwork 2. 


\section{Original artworks}

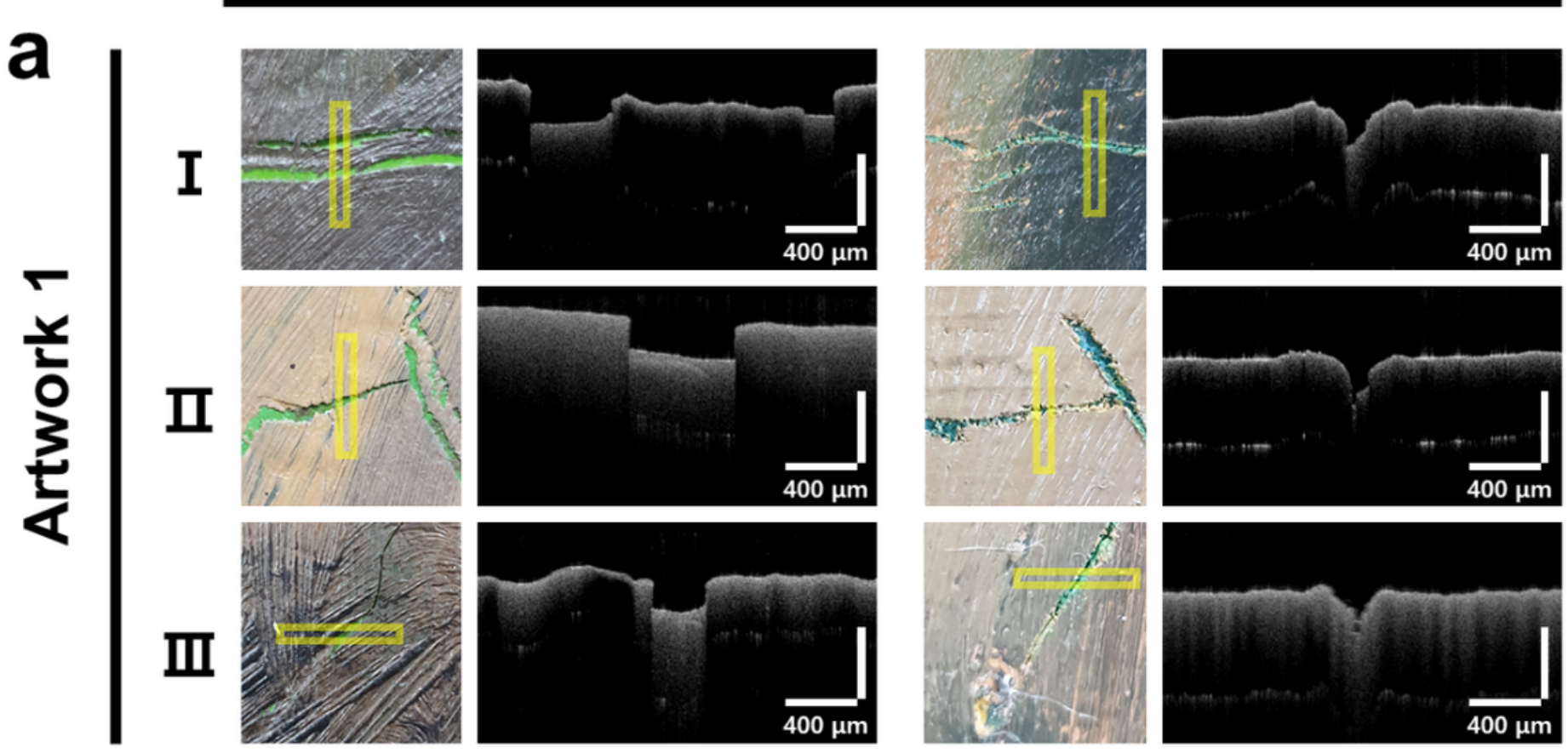

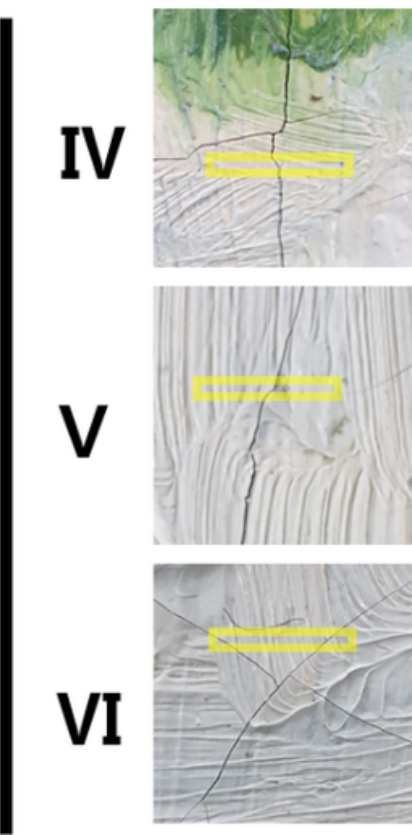
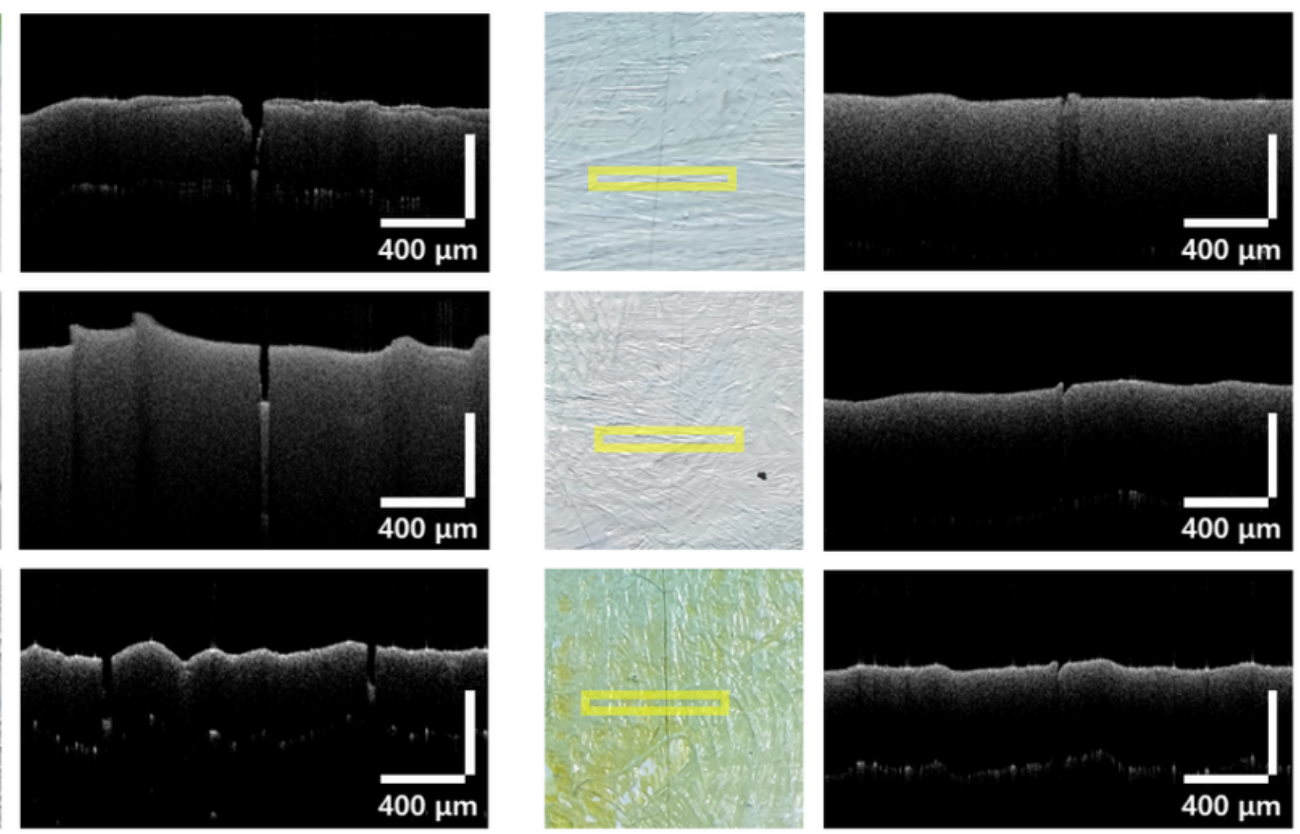

Figure 4

Comparison between the cross-sectional images obtained from the cracks in the original artworks and counterfeit cracks using the OCT system. (a) The cracks in Artwork 1 were caused by the small amount of medium in the upper layer but were formed in a wide rectangular shape. The forged cracks in Artwork 1 appear similar in depth to the original cracks, but unlike the original cracks, which are rectangular, they have an inverted triangle shape. (b) The original cracks in Artwork 2 occurred because the amount of 
medium in the lower layer was small, and the cracks were formed in a thin and very deep rectangular shape. Unlike the original cracks, the forged cracks in Artwork 2 are very shallow in depth.

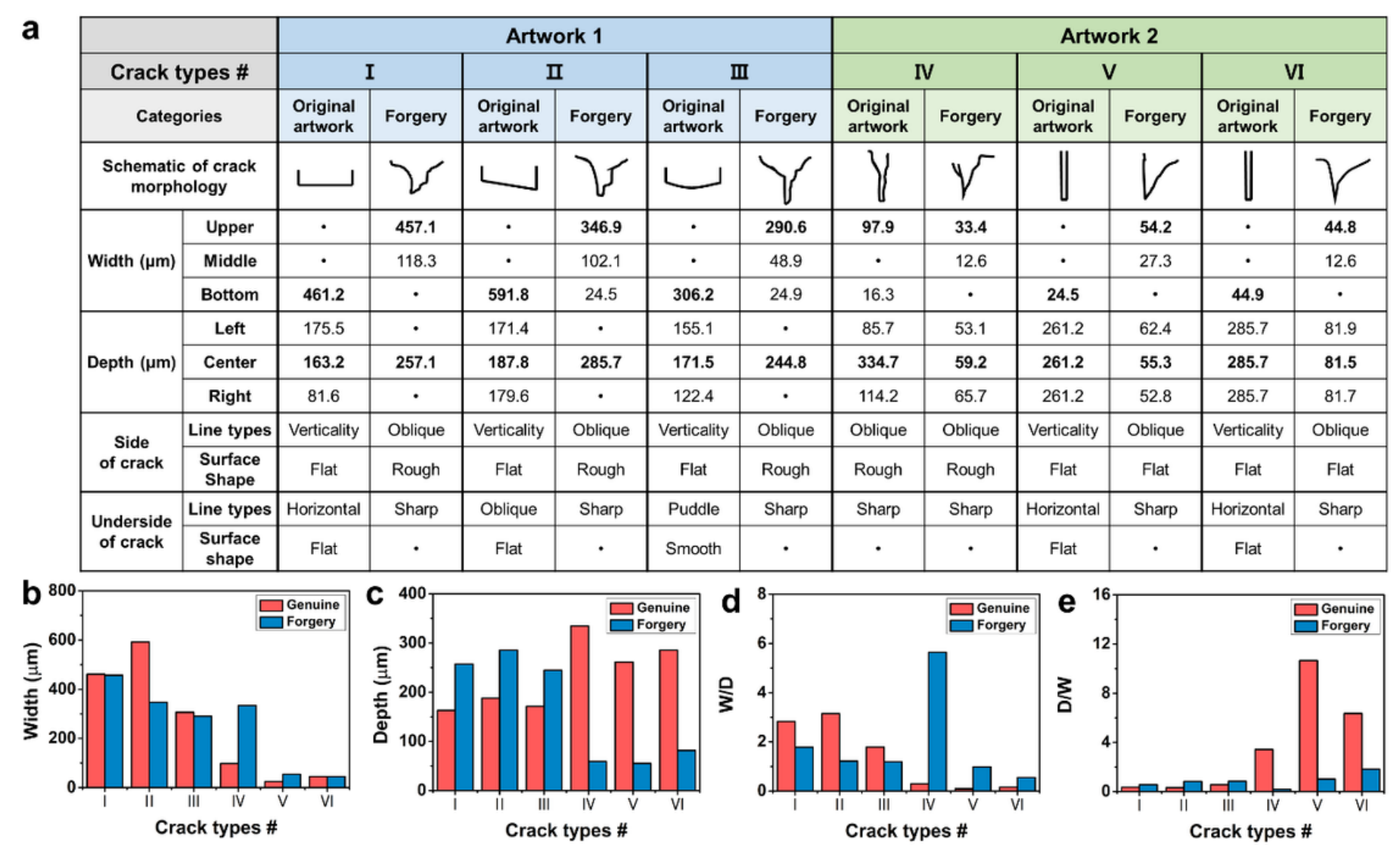

Figure 5

The cross-sectional shape, width and depth of each crack, the shape of the side of the crack, and the shape characteristics of the underside of the crack are described. (a) The crack widths and depths were subdivided into three types (upper, middle, bottom/left, center, and right), depending on the crosssectional shape of the cracks. The representative values of each measured parameter are separately indicated in bold letters. Among the dimensions of the original and forged cracks selected for analysis, the representative values were compared by (b) width, (c) depth, (d) width/depth, and (e) depth/width, respectively. 


\section{Original artwork}
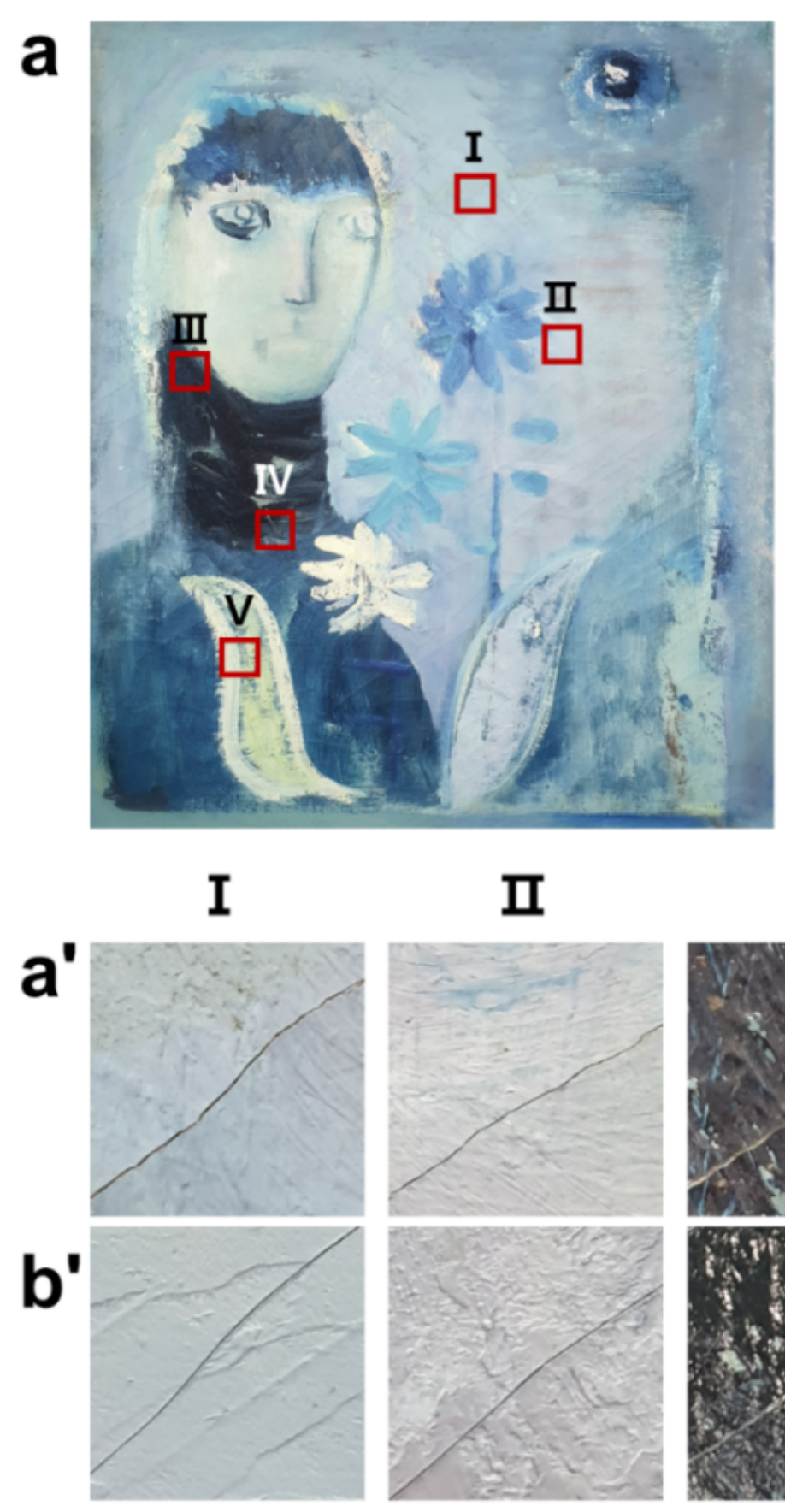
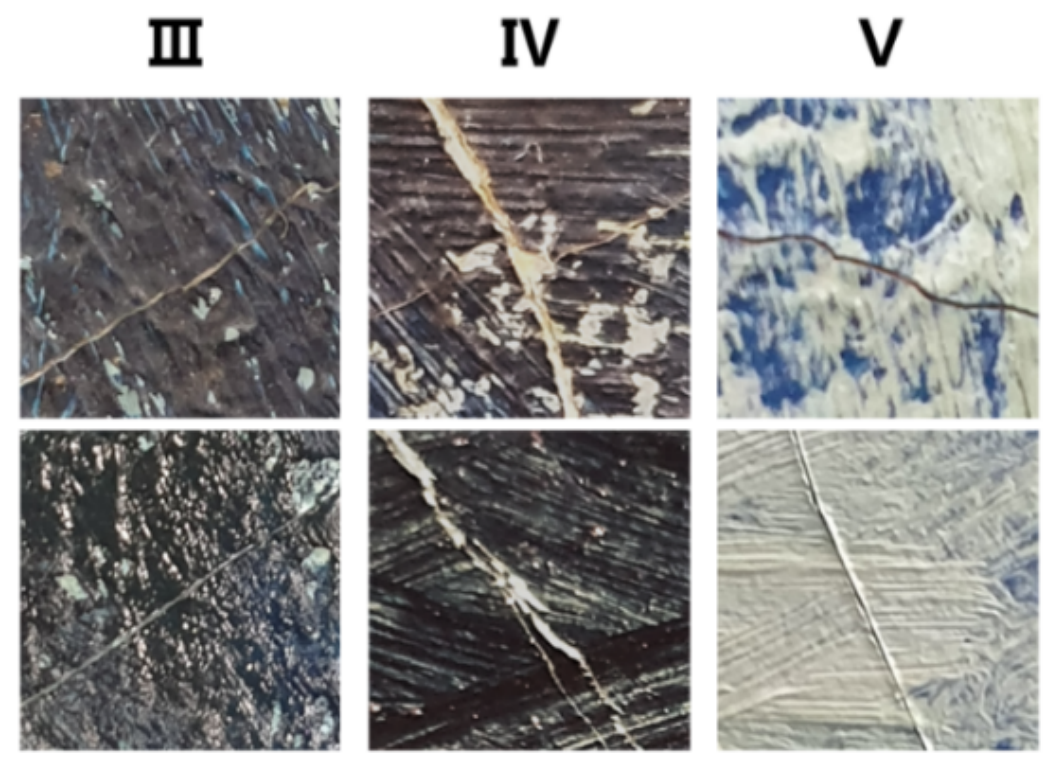

\section{Figure 6}

Images showing the location of cracks in (a) the original and (b) the forged oil paintings. The enlarged images of cracks selected as the OCT measurement area in ( $\left.a^{\prime}\right)$ the original artwork and ( $\left.b^{\prime}\right)$ the forged

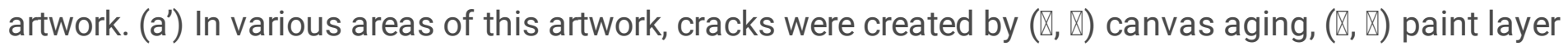

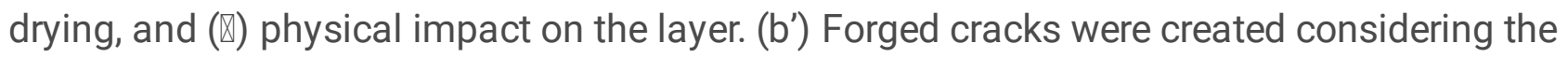
characteristics and causes of the original cracks. 


\section{Original artwork}
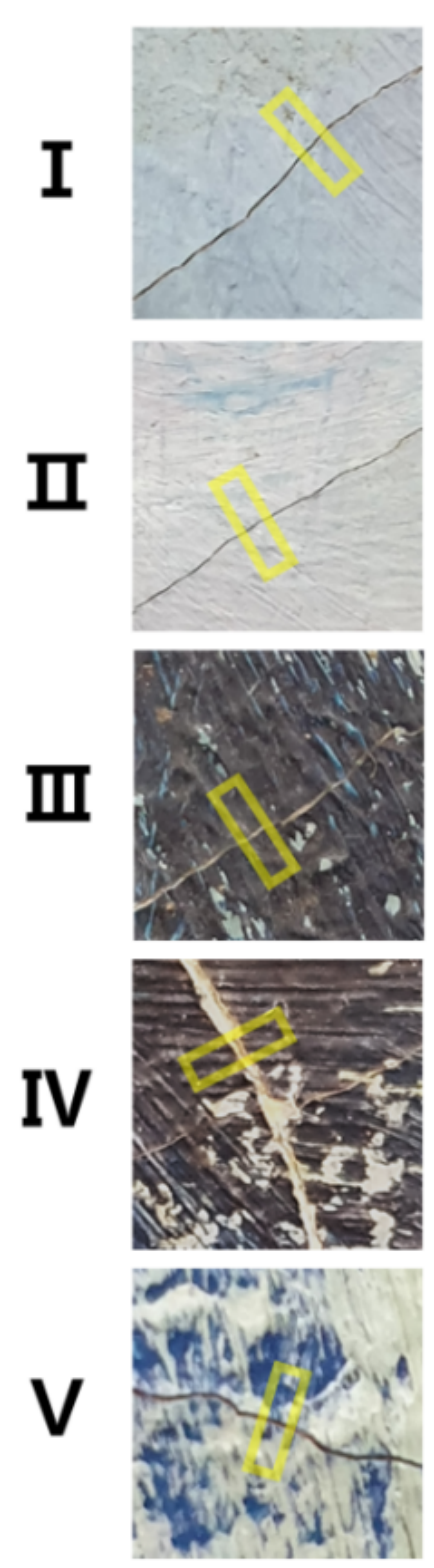
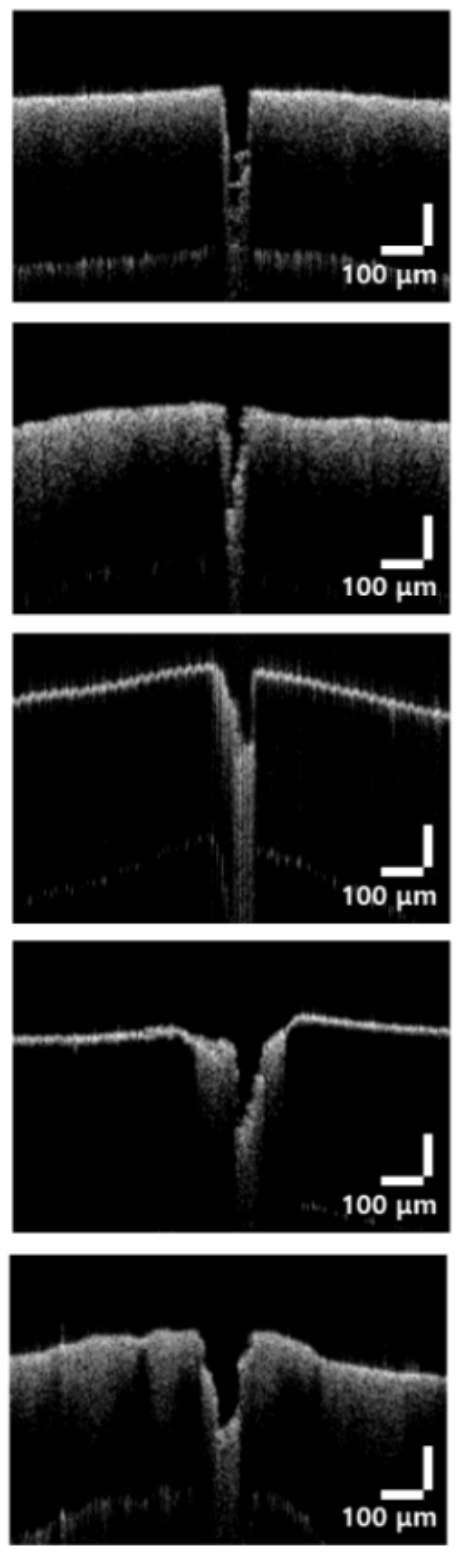

\section{Forgery}
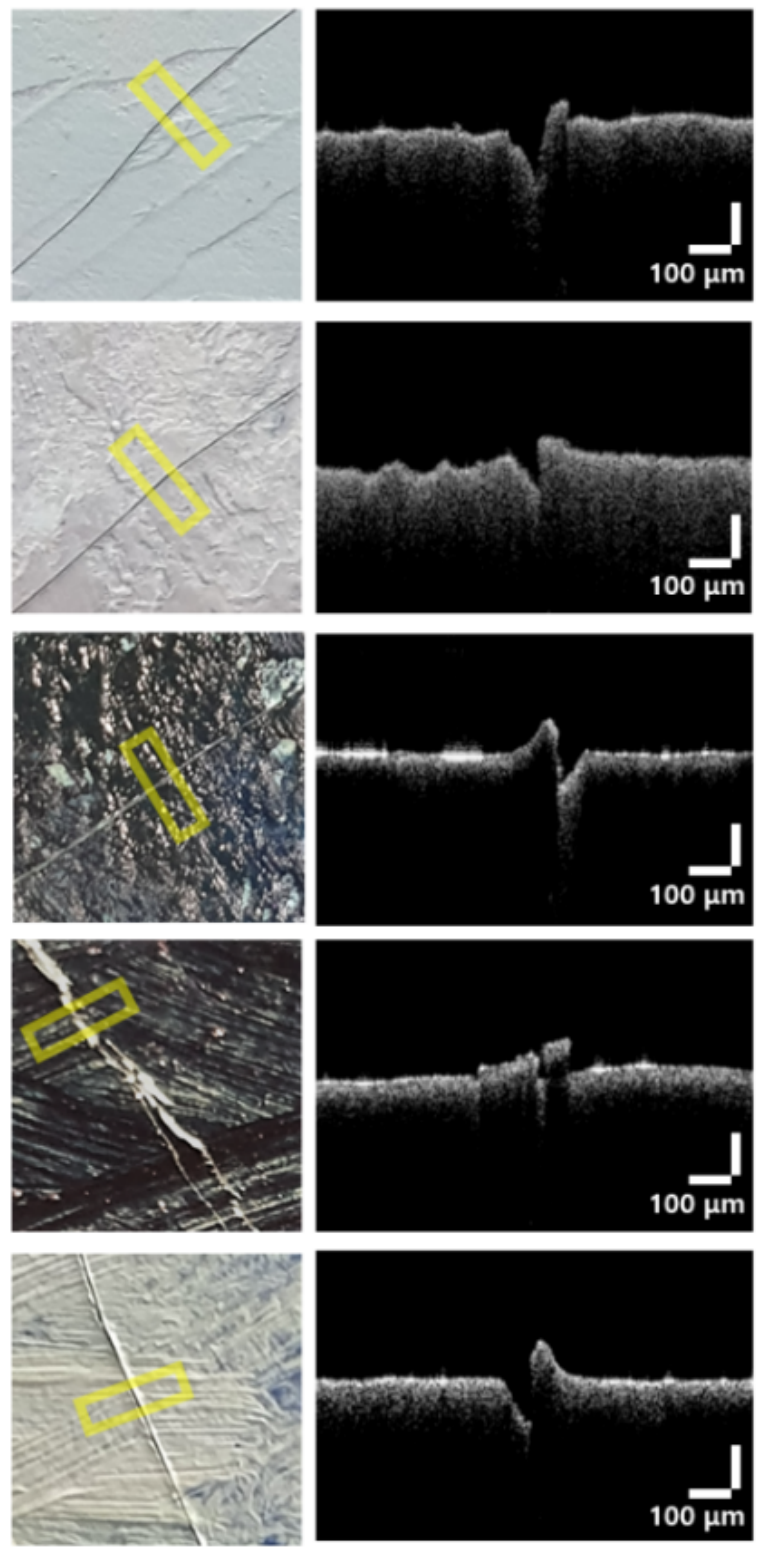

Figure 7

Comparison between OCT cross-sectional images of (a) an original and (b) forged artwork for art authentication. ( $\mathbb{\nabla})$ Cracks caused by the aging canvas made in areas covered in a thin layer of paint. ( $\mathbb{\nabla})$ Cracks caused by the aging canvas made in areas where the thickness of the paint layer was relatively thicker. ( $\nabla$ ) Cracks caused by the aging paint layer and made in areas with a thin layer. ( $($ ) Cracks made by applying a physical impact to the paint layer. (区) Cracks caused by the aging of the paint layer and made 
in a thick area of the paint layer. The side paint layer of the forged cracks protrudes and has a much shallower inverted triangle shape than the original cracks.

\section{Figure 8}

The shape characteristics of crack are described such as the width, depth, side shape, and underside of the cracks. (a) The widths and depths of cracks are subdivided into three types (upper, middle, bottom/left, center and right). The edges of the cracks show crack morphologies. The representative values of each measured parameter are indicated in bold and compared according to (b) width, (c) depth, (d) width/depth, and (e) depth/width.

\section{Supplementary Files}

This is a list of supplementary files associated with this preprint. Click to download.

- SupplementaryinformationArtcraquelure.docx 